\title{
Use of FDG-PET to guide dose prescription heterogeneity in stereotactic body radiation therapy for lung cancers with volumetric modulated arc therapy: a feasibility study
}

Bénédicte Henriques de Figueiredo ${ }^{1 *}$, Mikael Antoine ${ }^{1}$, Renaud Trouette ${ }^{2}$, Philippe Lagarde ${ }^{1}$, Adeline Petit ${ }^{1}$ Frédéric Lamare ${ }^{3}$, Mathieu Hatt ${ }^{4}$ and Philippe Fernandez ${ }^{3}$

\begin{abstract}
Background: The aim of this study was to assess if FDG-PET could guide dose prescription heterogeneity and decrease arbitrary location of hotspots in SBRT.

Methods: For three patients with stage I lung cancer, a CT-simulation and a FDG-PET were registered to define respectively the PTV $\mathrm{CT}$ and the biological target volume (BTV). Two plans involving volumetric modulated arc therapy (VMAT) and simultaneous integrated boost (SIB) were calculated. The first plan delivered $4 \times 12$ Gy within the PTV $\mathrm{C}_{C}$ and the second plan, with SIB, $4 \times 12$ Gy and $13.8 \mathrm{~Gy}$ (115\% of the prescribed dose) within the PTV $C$ and the BTV respectively. The Dmax-PTV $\mathrm{C}_{\text {T }}$ had to be inferior to $60 \mathrm{~Gy}$ (125\% of the prescribed dose). Plans were evaluated through

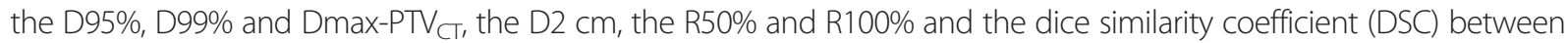
the isodose $115 \%$ and BTV. DSC allows verifying the location of the $115 \%$ isodose (ideal value $=1$ ).

Results: The mean PTV $V_{C T}$ and BTV were $36.7( \pm 12.5)$ and $6.5( \pm 2.2) \mathrm{cm}^{3}$ respectively. Both plans led to similar target coverage, same doses to the OARs and equivalent fall-off of the dose outside the PTV $\mathrm{C}_{\mathrm{C}}$. On the other hand, the location of hotspots, evaluated through the DSC, was improved for the SIB plans with a mean DSC of 0.31 and 0.45 for the first and the second plans respectively.
\end{abstract}

Conclusions: Use of PET to decrease arbitrary location of hotspots is feasible with VMAT and SIB for lung cancer.

Keywords: Stereotactic body radiation therapy, Lung cancer, Positron emission tomography, Volumetric modulated arc therapy

\section{Introduction}

Stereotactic body radiation therapy (SBRT) is considered as a standard treatment for patients with inoperable early stage lung cancer and leads to local control rates over $90 \%$ [1-4]. Historically, SBRT is delivered using a large number of non-overlapping treatment beams or arcs to create a sharper dose gradient between the target volume and the surrounding normal tissues. These delivery techniques result in large dose heterogeneity with a frequent dose prescription specified at the $80 \%$ (or lower)

\footnotetext{
* Correspondence: b.henriques@bordeaux.unicancer.fr

'Department of Radiotherapy, Institut Bergonié, 229, cours de l'Argonne,

F-33076 Bordeaux Cedex, France

Full list of author information is available at the end of the article
}

isodose and hotspots within the planning target volume (PTV). The recent ROSEL guidelines about SBRT recommend the maximum PTV dose to be between $110 \%$ and $140 \%$ of the prescription dose [5]. However, the location of these hotspots within the PTV is uncertain and uncontrolled by conventional SBRT delivery techniques.

Recent developments including volumetric modulated arc therapy (VMAT) with one or multiple arcs and varying openings of the multi-leaf collimator (MLC), dose rates and gantry speed, have resulted in more homogeneous dose distributions in SBRT [6]. However, clinical results of SBRT have historically been obtained with overdoses ranging from 120 to $140 \%$ inside the PTV, 
which possibly contribute the success of SBRT. In addition to delivering more homogeneous treatments, if used with simultaneous integrated boost (SIB), VMAT can also create a dose heterogeneity and controlled hotspots inside the PTV.

For non-small cell lung cancers (NSCLC), positron emission tomography (PET) with $\left[{ }^{18} \mathrm{~F}\right]$-Fluorodeoxyglucose (FDG) is frequently used to optimize radiotherapy for advanced stages with a better distinction between tumour and atelectasis, and a better detection of involved nodes. PET is not used for SBRT planning because the definition of the gross tumor volume (GTV) on computed tomography (CT) imaging in NSCLC stage I cases is not difficult. However, several studies have shown that FDG-PET could detect the sites at risk of failure inside GTV $[7,8]$. These observations led us to use FDG-PET to guide hotspots and dose prescription heterogeneity for NSCLC SBRT with VMAT.

For this purpose, a feasibility study was conducted by analyzing the dosimetries of three patients. For each patient, two VMAT plans were performed and compared: the first plan delivered SBRT using only CT data, whereas the second plan delivered SBRT using CT and FDG-PET data to guide dose prescription heterogeneity.

\section{Material and methods}

\section{Patient group}

Three patients, enrolled in a clinical trial assessing the impact of 4D-FDG-PET in radiotherapy for lung cancer, were selected for this dosimetric study. The study was approved by the institutional review board of the universitary hospital and the French health authority and patients gave written informed consent to participate. All patients were male with a median age of 70 years [62-72] and peripheral T1NOM0 NSCLC (two adenocarcinomas and one squamous cell carcinoma). The tumour was located in the left inferior lobe with attachment to the chest wall for patient 1 , in the middle lobe for patient 2 , and in the left upper lobe for patient 3 .

\section{Imaging}

For CT simulation, patients were in supine position with the arms above the head using an armrest. Data were acquired with a 3-mm slice in free breathing. No four dimensional (4D) acquisition was performed because 4D CT scan was not implemented in our department at the date of this study. For FDG-PET/CT, a 4D acquisition was performed in radiotherapy treatment position, $50 \mathrm{~min}$ after intravenously injection of $3.7 \mathrm{MBq} / \mathrm{Kg}$ of $\left[{ }^{18} \mathrm{~F}\right]$-FDG, on a PET/CT integrated system (Discovery RX, General Electric Medical System, Milwaukee, WI) using the Real-time Position Management (RPM) device (Varian $\left.{ }^{\circ}\right)$ and the "Motion Free" software (General Electric Medical System $^{\circ}$ ). The respiratory cycle was rebinned in six phases.

\section{Target volumes definition}

For the target volume definition, CT simulation and PET/ $\mathrm{CT}$ were registered using a rigid algorithm. The $\mathrm{GTV}_{\mathrm{CT}}$ was delineated manually on CT simulation using a parenchymal window ( -1000 and +200 Hounsfield Units). Patients 1 and 3 presented a non-mobile lesion (located in the upper lobe or attached to the chest), therefore a $5 \mathrm{~mm}$ isotropic margin was added to GTV to create $\mathrm{PTV}_{\mathrm{CT}}$. For patient 2, a $8 \mathrm{~mm}$ isotropic margin was added.

The Biological Target Volume (BTV) was defined on 4D-PET and corresponded to the sum of the six BTV delineated on the six respiratory-gated PET images. BTV was delineated on each PET image using the fuzzy locally adaptive Bayesian (FLAB) method (9), which is an automatic segmentation method. This statistic algorithm combined with a fuzzy measure is particularly adapted to noisy and blurry PET images, and has been validated on both simulated and clinical datasets for accuracy and robustness. Also, BTV included internal target volume (ITV) in its definition.

The lung-PTV, spinal cord, oesophagus, heart, trachea, brachial plexus and chest wall were outlined as organs at risk (OAR) on CT simulation.

\section{Dose prescription and treatment planning}

For each patient, two RapidArc plans (Varian Medical System $^{\circ}$ ) were calculated by the same radiophysicist using the Eclipse treatment planning software (Helios, Varian Medical System ${ }^{\circ}$ ) with the Analytic Anisotropic Algorithm v.10. For the first plan, the objective was to deliver 48 Gy in $\mathrm{PTV}_{\mathrm{CT}}$ in 4 fractions of $12 \mathrm{~Gy}$. For the second plan, the objective was to deliver $48 \mathrm{~Gy}$ in PTV $_{\text {CT }}$ and to guide hotspots in BTV with SIB. Both plans adopted the following ROSEL recommendations: $95 \%$ of the $\mathrm{PTV}_{\mathrm{CT}}$ had to receive at least $100 \%$ of the prescribed dose and $99 \%$ of the $\mathrm{PTV}_{\mathrm{CT}}$ at least $90 \%$ of the prescribed dose. The maximum dose within the PTV $_{\text {CT }}\left(\right.$ Dmax PTV $_{\text {CT }}$ ) had to be lower than $125 \%$ of the prescribed dose. Additional constraints included the $\mathrm{D} 2 \mathrm{~cm}$ (maximal dose at $2 \mathrm{~cm}$ of the $\mathrm{PTV}_{\mathrm{CT}}$ ), the $\mathrm{R} 50 \%$ and $\mathrm{R} 100 \%$ (ratios between $\mathrm{PTV}_{\mathrm{CT}}$ and the $50 \%$ and $100 \%$ isodoses, respectively) allowing to verify the rapid fall-off of the dose outside the PTV $\mathrm{PT}_{\mathrm{CT}}$. The OARs dose constraints were in accordance with the RTOG 0915 trial. The Dmax of the spinal cord, the brachial plexus, the oesophagus, the heart and the trachea were inferior to 26 , 27.2, 30, 34 and $34.8 \mathrm{~Gy}$, respectively. For the chest wall, the dose to one $\mathrm{cm}^{3}$ (D1cc) was kept below 32Gy.

RapidArc plans were performed with three $180^{\circ}$ coplanar arcs using $6 \mathrm{MV}$ photons at a maximum dose rate of 600 monitor units (MUs) per minute. Collimator angles of 15 , 30 and $330^{\circ}$ were used to minimize the tongue-and-groove effect. For the first plan, 12 Gy per fraction were prescribed at the barycentre of the $\mathrm{PTV}_{\mathrm{CT}}$. For the second plan, $12 \mathrm{~Gy}$ 
and $13.8 \mathrm{~Gy}$ (115\% of the prescribed dose) were prescribed with SIB in PTV $\mathrm{CT}_{\mathrm{C}}$ and BTV, respectively.

\section{Dosimetric analysis}

In order to assess hotspots, we calculated the volume of the $115 \%$ isodose $\left(\mathrm{V}_{\text {iso115\% }}\right)$ and the Dice Similarity Coefficient (DSC). DSC allows to verify the location of the $115 \%$ isodose, and was defined as follows: $\mathrm{DSC}=\left(\mathrm{V}_{\text {iso115\% }} \cap \mathrm{V}_{\mathrm{BTV}}\right) /$ $\left(\mathrm{V}_{\text {iso115\% }} \cup \mathrm{V}_{\mathrm{BTV}}\right)$. Ideally, DSC is equal to 1 .

\section{Results}

The mean PTV $_{\mathrm{CT}}$ and BTV were $36.7( \pm 12.5)$ and $6.5( \pm 2.2) \mathrm{cm}^{3}$, respectively. For both plans, ROSEL recommendations were respected. The SIB technique (plan 2) led to similar target coverage than that obtained with conventional prescription on $\mathrm{PTV}_{\mathrm{CT}}$ (plan 1): the mean D95\%, D99\% and the Dmax of the $\mathrm{PTV}_{\mathrm{CT}}$ were $103.7 \% \pm 1.3,101.6 \% \pm 2.1$ and $123.5 \% \pm 1.9$ without SIB, versus $103.7 \% \pm 2.4,101.3 \% \pm 2.1$ and $124.4 \% \pm 0.3$ with SIB. The rapid fall-off of the dose outside the PTV $\mathrm{CT}_{\mathrm{T}}$ was equivalent for both plans: the mean D2cm, R50\% and $\mathrm{R} 100 \%$ were $62.4 \% \pm 3.9,4.6 \% \pm 0.7$ and $1.1 \% \pm 0.08$ without SIB, versus $66.2 \% \pm 3.9,4.7 \% \pm 0.9$ and $1.09 \% \pm 0.1$ with SIB.

For both plans, constraints of doses to the OARs were respected, except for the dose to the chest wall for patient 1 , who presented a tumour attached to the rib.

To assess the ability of the SIB to guide hotspots, we evaluated the volume of the $115 \%$ isodose and the location of this isodose through the analysis of the DSC (Table 1). The Viso115\% was more important in plan 2, with a $33 \%$ and $16 \%$ increase for patients 1 and 3, respectively. On the contrary, for patient 2 the Viso115\% was bigger in the plan 1 with a difference of $85 \%$. Analysis on the location of the $115 \%$ isodose revealed that SIB technique improves DSC with a mean of 0.31 for plan 1 and 0.45 for plan 2.. SIB technique's ability to guide hotspots in the BTV and to decrease arbitrary hotspots is illustrated in Figure 1.

\section{Discussion}

One limitation of this study is the lack of 4D-CT simulation, which impedes a good estimation of the ITV. An isotropic margin was also added automatically around the

Table 1 The $115 \%$ isodose and the dice similarity coefficient (DSC)

\begin{tabular}{|c|c|c|c|c|}
\hline \multirow[t]{2}{*}{ Patient } & \multicolumn{2}{|c|}{ Viso115\% $\left(\mathrm{cm}^{3}\right)$} & \multicolumn{2}{|c|}{ DSC BTV-Viso115\% (\%) } \\
\hline & Plan 1 & Plan 2-SIB & Plan 1 & Plan 2-SIB \\
\hline 1 & 14 & 18.6 & 0.26 & 0.45 \\
\hline 2 & 22.2 & 12 & 0.21 & 0.4 \\
\hline 3 & 10 & 11.6 & 0.45 & 0.51 \\
\hline Mean ( \pm SD) & $15.4 \pm 6.2$ & $14.1 \pm 3.9$ & $0.31 \pm 0.13$ & $0.45 \pm 0.06$ \\
\hline
\end{tabular}

$\mathrm{GTV}_{\mathrm{CT}}$, probably leading to an overestimation of the $\mathrm{PTV}_{\mathrm{CT}}$. The present investigation, however, does not focus on target volume definition, but rather on the use of PET and VMAT to control and guide hotspots in SBRT. In this respect, the study demonstrates that it is feasible to guide dose prescription heterogeneity on FDG-PET data with VMAT and SIB. Indeed, plans 1 and 2 respected conventional dosimetric criteria of SBRT equally (D95\% PTV $\mathrm{CT}_{\mathrm{CT}}$, 99\% $\mathrm{PTV}_{\mathrm{CT}}$, Dmax $\mathrm{PTV}_{\mathrm{CT}}$, $\mathrm{D} 2 \mathrm{~cm}, \mathrm{R} 50 \%$ and $\mathrm{R} 100 \%)$ and imparted equivalent doses to the OARs. In contrast, better DSC was computed with plan 2. In this study, we chose to perform a SIB with a dose of $115 \%$ of the prescribed dose, because the BTV defined on 4D-FDG-PET is relatively large considering the ITV. FDG-PET was used because its ability to detect sites of relapse after radiotherapy is recognized. After conventional fractionated radiotherapy treatments, relapses are often located inside the area of highest uptake on baseline FDG-PET scan $[7,8]$. That's why some authors try to increase the radiation dose inside this BTV. Our purpose was based on this idea and was to integrate data of FDG-PET in SBRT focusing hotspots on BTV. Other PET tracers like hypoxia or proliferation tracers could be of interest to individualize more specific BTVs. These BTVs issued from more specific tracers are often smaller than those defined with FDG. The choice of isodose $(115,120 \%$ or higher) to focus hotspots could also vary depending on the tracer and volume of BTV. Whatever the choice of the tracer, an important difficulty stays the choice of the method of PET images segmentation. Many automatic segmentation methods are described in the literature and in the absence of a confrontation with gold standards or surgical specimens, it's impossible to promote one method. More complex segmentation methods as gradient based or stochastic methods seem more robust than thresholding methods based on SUV $[9,10]$.

VMAT is particularly interesting to plan and deliver SBRT. The number of studies concerned with SBRT and VMAT has incredibly increased these last years. Several dosimetric comparisons confirmed an important reduction in treatment time compared to other delivery techniques like three-dimensional conformal radiotherapy, Cyberknife or Tomotherapy [6,11-14] as well as a reduction of patient movements caused by discomfort and a reduction of the consecutive intrafraction motion. VMAT can achieve better conformity, sharper dose fall-off outside the PTV and lower dose to normal lung [12-15].

However, concerns about use of modulation therapy for SBRT for tumors subject to respiratory motion have been previously highlighted with the "interplay effect" [16]. This effect describes the interplay between MLC motion and tumour motion with risk of hotspots or cold 

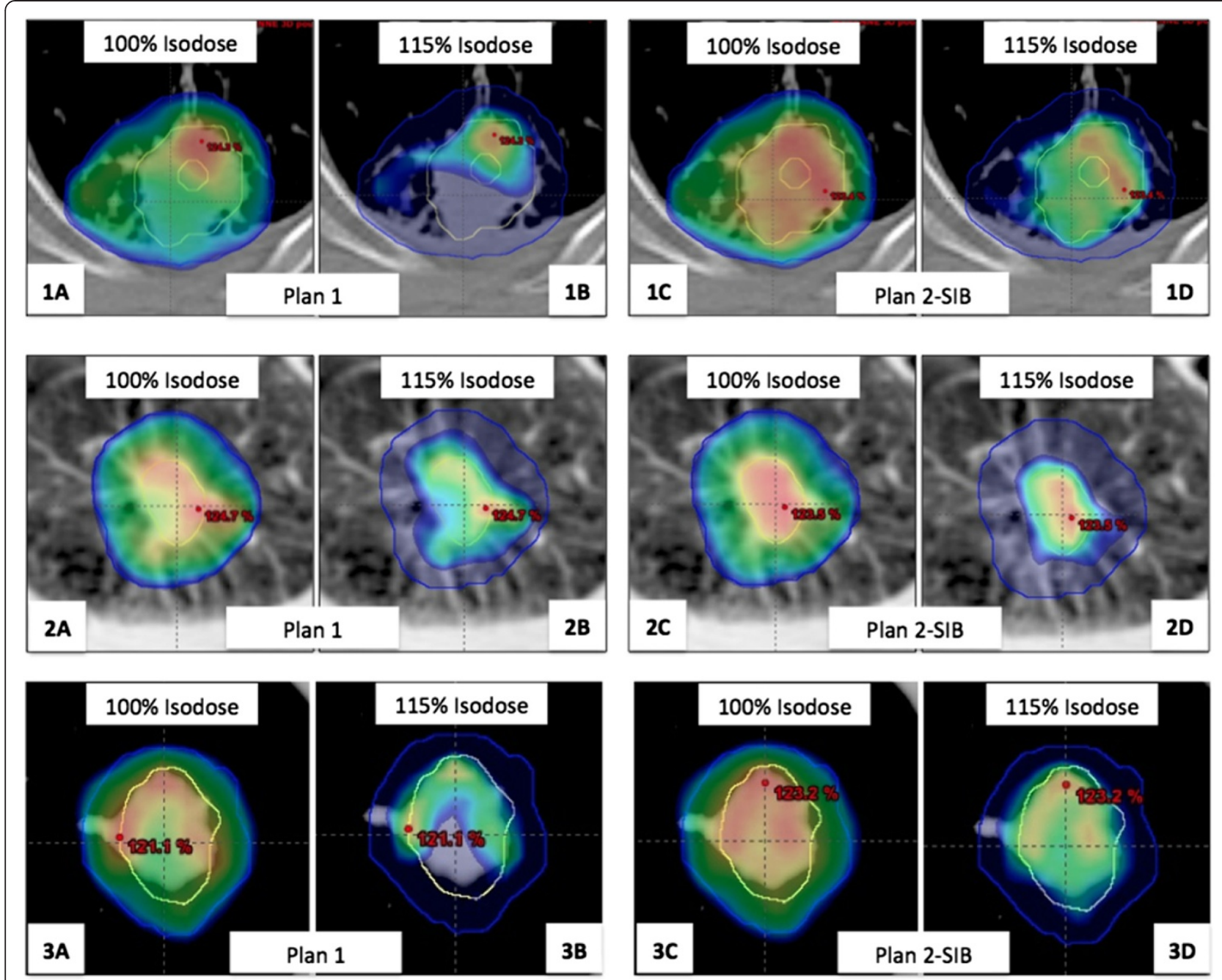

Figure 1 Comparison of both plans for the three patients: plans 1 in (A) and (B) and plans 2 with SIB in (C) and (D). PTV $V_{C T}$ is shown in blue and BTV in yellow. With both techniques, the target coverage of the PTV $\mathrm{CT}_{\mathrm{T}}$ with the $100 \%$ isodose is adequate but the $115 \%$ isodose is more focused on the BTV with the SIB technique.

spots inside the tumor. Recent series have investigated the impact of tumour motion on 4D dose calculation for lung SBRT and found a negligible interplay effect, with differences between 3D and 4D calculations around 1\% $[17,18]$. Several reasons may explain this negligible effect. First, since high doses per fraction are delivered during SBRT, most VMAT segments receive a large number of MUs with a long segment delivery time (on the order of the breathing period). Moreover, modulation for lung SBRT is low with few OARs. Also, most segments contain an aperture shape conformed to the PTV and few segments have leaves blocking a part of the tumor. In the same way, the gantry speed is in general low to be able to deliver a large number of MUs at each segment. Few clinical studies about modulation intensity for lung SBRT are published but results of the Mayo clinic with IMRT [19], of Yamashita et al. [20] with VMAT or Navarria et al. [15] with VMAT unflattened beams (FFF) are good with excellent local control.

\section{Conclusion}

Use of PET to guide dose prescription heterogeneity with VMAT and SIB for lung cancer is feasible and seems promising. These preliminary results have to be confirmed with more patients and confronted to a clinical study. Moreover, other PET tracers more specific than FDG could be evaluated for this dose painting.

\section{Competing interests}

The authors declare that they have no competing interests. 


\section{Authors' contributions}

$\mathrm{BH}$ and MA carried out the planification of the dosimetric study. MH and FL participated in the segmentation of PET images. RT, PL, AP and PF drafted the manuscript.

\section{Author details}

'Department of Radiotherapy, Institut Bergonié, 229, cours de l'Argonne, F-33076 Bordeaux Cedex, France. ${ }^{2}$ Department of Radiotherapy, Hospital Haut-Lévêque, CHRU Bordeaux, Bordeaux F-33076, France. ${ }^{3}$ Department of Nuclear Medicine, Hospital Pellegrin, CHRU Bordeaux, Bordeaux F-33076, France. ${ }^{4}$ LaTIM INSERM, U1101 Brest, France.

Received: 30 September 2014 Accepted: 14 December 2014 Published online: 23 December 2014

\section{References}

1. Baumann P, Nyman J, Hoyer M, Wennberg B, Gagliardi G, Lax I, Drugge N, Ekberg L, Friesland S, Johansson K-A, Lund J-A, Morhed E, Nilsson K, Levin N, Paludan M, Sederholm C, Traberg A, Wittgren L, Lewensohn R: Outcome in a prospective phase II trial of medically inoperable stage I non-small-cell lung cancer patients treated with stereotactic body radiotherapy. J Clin Oncol Off J Am Soc Clin Oncol 2009, 27:3290-3296.

2. Brock J, Ashley S, Bedford J, Nioutsikou E, Partridge M, Brada M: Review of hypofractionated small volume radiotherapy for early-stage non-small cell lung cancer. Clin Oncol R Coll Radiol G B 2008, 20:666-676.

3. Fakiris AJ, McGarry RC, Yiannoutsos $C T$, Papiez L, Williams M, Henderson MA, Timmerman R: Stereotactic body radiation therapy for early-stage non-small-cell lung carcinoma: four-year results of a prospective phase II study. Int J Radiat Oncol Biol Phys 2009, 75:677-682.

4. Lagerwaard FJ, Haasbeek CJA, Smit EF, Slotman BJ, Senan S: Outcomes of risk-adapted fractionated stereotactic radiotherapy for stage I non-small-cell lung cancer. Int J Radiat Oncol Biol Phys 2008, 70:685-692.

5. Hurkmans CW, Cuijpers JP, Lagerwaard FJ, Widder J, van der Heide UA, Schuring D, Senan S: Recommendations for implementing stereotactic radiotherapy in peripheral stage IA non-small cell lung cancer: report from the quality assurance working party of the randomised phase III ROSEL study. Radiat Oncol Lond Engl 2009, 4:1

6. Verbakel WFAR, Senan S, Cuijpers JP, Slotman BJ, Lagerwaard FJ: Rapid delivery of stereotactic radiotherapy for peripheral lung tumors using volumetric intensity-modulated arcs. Radiother Oncol I Eur Soc Ther Radiol Oncol 2009, 93:122-124.

7. Abramyuk A, Tokalov S, Zöphel K, Koch A, Szluha Lazanyi K, Gillham C, Herrmann T, Abolmaali N: Is pre-therapeutical FDG-PET/CT capable to detect high risk tumor subvolumes responsible for local failure in non-small cell lung cancer? Radiother Oncol J Eur Soc Ther Radiol Oncol 2009, 91:399-404.

8. Aerts HJWL, van Baardwijk AAW, Petit SF, Offermann C, van Loon J, Houben R, Dingemans A-MC, Wanders R, Boersma L, Borger J, Bootsma G, Geraedts W, Pitz C, Simons J, Wouters BG, Oellers M, Lambin P, Bosmans G, Dekker ALAJ, De Ruysscher D: Identification of residual metabolic-active areas within individual NSCLC tumours using a pre-radiotherapy (18)FluorodeoxyglucosePET-CT scan. Radiother Oncol J Eur Soc Ther Radiol Oncol 2009, 91:386-392.

9. Hatt M, Cheze le Rest C, Turzo A, Roux C, Visvikis D: A fuzzy locally adaptive Bayesian segmentation approach for volume determination in PET. IEEE Trans Med Imaging 2009, 28:881-893.

10. Wanet M, Lee JA, Weynand B, De Bast M, Poncelet A, Lacroix V, Coche E, Grégoire V, Geets X: Gradient-based delineation of the primary GTV on FDG-PET in non-small cell lung cancer: a comparison with threshold-based approaches, CT and surgical specimens. Radiother Oncol $J$ Eur Soc Ther Radiol Oncol 2011, 98:117-125.

11. Ong CL, Verbakel WFAR, Cuijpers JP, Slotman BJ, Lagerwaard FJ, Senan S: Stereotactic radiotherapy for peripheral lung tumors: a comparison of volumetric modulated arc therapy with 3 other delivery techniques. Radiother Oncol J Eur Soc Ther Radiol Oncol 2010, 97:437-442.

12. Zhang GG, Ku L, Dilling TJ, Stevens CW, Zhang RR, Li W, Feygelman V: Volumetric modulated arc planning for lung stereotactic body radiotherapy using conventional and unflattened photon beams: a dosimetric comparison with 3D technique. Radiat Oncol Lond Engl 2011, 6:152.

13. Holt A, van Vliet-Vroegindeweij C, Mans A, Belderbos JS, Damen EMF: Volumetric-modulated arc therapy for stereotactic body radiotherapy of lung tumors: a comparison with intensity-modulated radiotherapy techniques. Int J Radiat Oncol Biol Phys 2011, 81:1560-1567.

14. McGrath SD, Matuszak MM, Yan D, Kestin LL, Martinez AA, Grills IS: Volumetric modulated arc therapy for delivery of hypofractionated stereotactic lung radiotherapy: A dosimetric and treatment efficiency analysis. Radiother Oncol J Eur Soc Ther Radiol Oncol 2010, 95:153-157.

15. Navarria P, Ascolese AM, Mancosu P, Alongi F, Clerici E, Tozzi A, Iftode C, Reggiori G, Tomatis S, Infante M, Alloisio M, Testori A, Fogliata A, Cozzi L, Morenghi E, Scorsetti M: Volumetric modulated arc therapy with flattening filter free (FFF) beams for stereotactic body radiation therapy (SBRT) in patients with medically inoperable early stage non small cell lung cancer (NSCLC). Radiother Oncol J Eur Soc Ther Radiol Oncol 2013, 107:414-418.

16. Bortfeld T, Jiang SB, Rietzel E: Effects of motion on the total dose distribution. Semin Radiat Oncol 2004, 14:41-51.

17. Ong C, Verbakel WFAR, Cuijpers JP, Slotman BJ, Senan S: Dosimetric impact of interplay effect on RapidArc lung stereotactic treatment delivery. Int J Radiat Oncol Biol Phys 2011, 79:305-311.

18. Rao M, Wu J, Cao D, Wong T, Mehta V, Shepard D, Ye J: Dosimetric impact of breathing motion in lung stereotactic body radiotherapy treatment using intensity modulated radiotherapy and volumetric modulated arc therapy [corrected]. Int J Radiat Oncol Biol Phys 2012, 83:e251-e256.

19. Videtic GMM, Stephans K, Reddy C, Gajdos S, Kolar M, Clouser E, Djemil T: Intensity-modulated radiotherapy-based stereotactic body radiotherapy for medically inoperable early-stage lung cancer: excellent local control. Int I Radiat Oncol Biol Phys 2010, 77:344-349.

20. Yamashita H, Takahashi W, Haga A, Kida S, Saotome N, Nakagawa K: Stereotactic body radiotherapy for small lung tumors in the university of Tokyo hospital. BioMed Res Int 2014, 2014:136513.

\section{Submit your next manuscript to BioMed Central and take full advantage of:}

- Convenient online submission

- Thorough peer review

- No space constraints or color figure charges

- Immediate publication on acceptance

- Inclusion in PubMed, CAS, Scopus and Google Scholar

- Research which is freely available for redistribution 